\title{
Delays effects in dynamical systems and networks: analysis and control interpretations
}

\author{
Wim Michiels
}

\begin{abstract}
Time-delays are important components of many systems from engineering, economics and the life sciences, due to the fact that the transfer of material, energy and information is mostly not instantaneous. They appear for instance as computation and communication lags, they model transport phenomena and hereditary and they arise as feedback delays in control loops. The aim of the chapter is to present a guided tour on stand-alone and interconnected systems with delays, thereby explaining some important qualitative properties. The focus rather lies on the main ideas as technical details are avoided. Different mechanisms in which delays can interact with the system are outlined, with the emphasis on the effects of delays on stability. It is clarified how these mechanisms affect control design problems. Not only limitations induced by delays in control loops are discussed, but also opportunities to use delays in the construction of controllers. Finally, extensions of these results toward networks of interconnected dynamical systems are discussed, with the focus on relative stability problems, in particular the synchronization problem.
\end{abstract}

\section{Introduction}

Time-delays are important components of many systems from engineering, economics and the life sciences, due to the fact that the transfer of material, energy and information is mostly not instantaneous. They appear, for instance, as computation and communication lags, they model transport phenomena and heredity and they arise as feedback delays in control loops. An overview of applications, ranging from traffic flow control and lasers with

Wim Michiels

Department of Computer Science, KU Leuven, Celestijnenlaan 200A, 3001 Heverlee, Belgium, e-mail: Wim.Michiels@cs.kuleuven.be 
phase-conjugate feedback, over (bio)chemical reactors and cancer modeling, to control of communication networks and control via networks is included in $[12]$.

The presence of time-delays in dynamical systems may induce complex behavior, and this behavior is not always intuitive. Even if a system's equation is scalar oscillations may occur. Time-delays in control loops are usually associated with degradation of performance and robustness, but, at the same time, there are situations where time-delays are used as controller parameters.

The aim of this chapter is to describe some important properties of control systems subjected to time-delays and to outline principles behind analysis and synthesis methods. Throughout the text, the results will be illustrated by means of the scalar system

$$
\dot{x}(t)=u(t-\tau)
$$

which, controlled with instantaneous state feedback, $u(t)=-k x(t)$, leads to the closed-loop system

$$
\dot{x}(t)=-k x(t-\tau) .
$$

Although this didactic example is extremely simple, we shall see that its dynamics are already very rich and shed a light on delay effects in control loops.

In some works the analysis of (2) is called the hot shower problem, as it can be interpreted as a (over)simplified model for a human adjusting the temperature in a shower: $x(t)$ then denotes the difference between the water temperature and the desired temperature as felt by the person, the term $-k x(t)$ models the reaction of the person by further opening or closing taps, and the delay is due to the propagation with finite speed of the water in the ducts.

The structure of the chapter is as follows. In Section 2 we outline fundamental properties of time-delays systems. In Section 3 we discuss spectral properties of linear time-delay systems. In Section 4 we discuss limitations of delays in control loops, but also opportunities of using delays for control purposes, and in Section 5 we make the leap to networks of interconnected systems, focusing on the synchronization problem. A short version of Sections 2-4 appeared in [7].

\section{Basic properties of time-delay systems}

\subsection{Functional differential equation}

We focus on a model for a time-delay system described by 


$$
\dot{x}(t)=A_{0} x(t)+A_{1} x(t-\tau), \quad x(t) \in \mathbb{R}^{n} .
$$

This is an example of a functional differential equation (FDE) of retarded type. The term FDE stems from the property that the right-hand side can be interpreted as a functional evaluated at a piece of trajectory. The term retarded expresses that the right hand side does not explicitly depend on $\dot{x}$.

As a first difference with an ordinary differential equation, the initial condition of (3) at $t=0$ is a function $\phi$ from $[-\tau, 0]$ to $\mathbb{R}^{n}$. For all $\phi \in \mathcal{C}\left([-\tau, 0], \mathbb{R}^{n}\right)$, where $\mathcal{C}\left([-\tau, 0], \mathbb{R}^{n}\right)$ is the space of continuous functions mapping the interval $[-\tau, 0]$ into $\mathbb{R}^{n}$, a forward solution $x(\phi)$ exists and is uniquely defined. In Figure 1, a solution of the scalar system

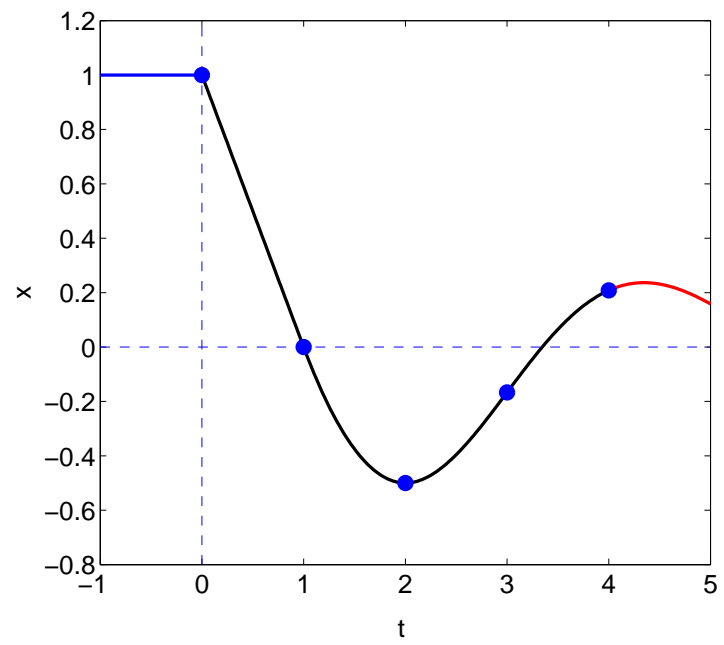

Fig. 1 Solution of (2) for $\tau=1, k=1$ and initial condition $\phi \equiv 1$.

(2) is shown. The discontinuity in the derivative at $t=0$ stems from $A_{0} \phi(0)+A_{1} \phi(-\tau) \neq \lim _{\theta \rightarrow 0-} \dot{\phi}(\theta)$. Due to the smoothing property of an integrator, however, at $t=n \in \mathbb{N}$, the discontinuity will only be present in the $(n+1)$-th derivative. This illustrates a second property of functional differential equations of retarded type: solutions become smoother as time evolves. As a third major difference with ODEs, backward continuation of solutions is not always possible [8].

The extension of methods for time-integration (time stepping, simulation) from ordinary to delay differential equations naturally follows from the properties of solutions sketched above: the "history" of the solution should be taken into account in the time-stepper, and special attention should be paid to so-called break points, where the solution is non-smooth. A key reference is the book [1]. 


\subsection{Reformulation in a first-order form}

The state of system (3) at time $t$ is the minimal information needed to continue the solution, which, once again, boils down to a function segment $x_{t}(\phi)$ where $x_{t}(\phi)(\theta)=x(t+\theta), \theta \in[-\tau, 0]$ (in Figure 1 the function $x_{t}$ is shown in red for $t=5)$. This suggests that (3) can be reformulated as a standard ordinary differential equation over the infinite-dimensional space $\mathcal{C}\left([-\tau, 0], \mathbb{R}^{n}\right)$. This equation takes the form

$$
\frac{d}{d t} z(t)=\mathcal{A} z(t), z(t) \in \mathcal{C}\left([-\tau, 0], \mathbb{R}^{n}\right)
$$

where operator $\mathcal{A}$ is given by

$$
\begin{aligned}
& \mathcal{D}(\mathcal{A})=\left\{\begin{array}{r}
\phi \in \mathcal{C}\left(\left[-\tau_{m}, 0\right], \mathbb{R}^{n}\right): \dot{\phi} \in \mathcal{C}\left(\left[-\tau_{m}, 0\right], \mathbb{R}^{n}\right), \\
\dot{\phi}(0)=A_{0} \phi(0)+A_{1} \phi(-\tau)
\end{array}\right\}, \\
& \mathcal{A} \phi=\frac{d \phi}{d \theta} .
\end{aligned}
$$

The relation between solutions of (3) and (4) is given by $z(t)(\theta)=x(t+$ $\theta), \theta \in[-\tau, 0]$. Note that all system information is concentrated in the nonlocal boundary condition describing the domain of $\mathcal{A}$. The representation (4) is closely related to a description by an advection PDE with a non-local boundary condition [5].

\subsection{Asymptotic growth rate of solutions and stability}

The reformulation of (3) into the standard form (4) allows us to define stability notions and to generalize the stability theory for ordinary differential equations in a straightforward way, with the main change that the state space is $\mathcal{C}\left([-\tau, 0], \mathbb{R}^{n}\right)$. For example, the null solution of $(3)$ is exponentially stable if and only if there exist constants $C>0$ and $\gamma>0$ such that

$$
\forall \phi \in \mathcal{C}\left(\left[-\tau_{m}, 0\right], \mathbb{R}^{n}\right) \quad\left\|x_{t}(\phi)\right\|_{s} \leq C e^{-\gamma t}\|\phi\|_{s}
$$

where $\|\cdot\|_{s}$ is the supremum norm, $\|\phi\|_{s}=\sup _{\theta \in[-\tau, 0]}\|\phi(\theta)\|_{2}$. As the system is linear asymptotic and exponential stability are equivalent. A direct generalization of Lyapunov's second method yields:

Theorem 1. The null solution of linear system (3) is asymptotically stable if there exist a continuous functional $V: \mathcal{C}\left([-\tau, 0], \mathbb{R}^{n}\right) \rightarrow \mathbb{R}$ (a socalled Lyapunov-Krasovskii functional) and continuous nondecreasing functions $u, v, w: \mathbb{R}^{+} \rightarrow \mathbb{R}^{+}$with

$$
u(0)=v(0)=w(0)=0 \text { and } u(s)>0, v(s)>0, w(s)>0 \text { for } s>0,
$$


such that for all $\phi \in \mathcal{C}\left([-\tau, 0], \mathbb{R}^{n}\right)$

$$
u\left(\|\phi\|_{s}\right) \leq V(\phi) \leq v\left(\|\phi(0)\|_{2}\right), \dot{V}(\phi) \leq-w\left(\|\phi(0)\|_{2}\right),
$$

where

$$
\dot{V}(\phi)=\limsup _{h \rightarrow 0+} \frac{1}{h}\left[V\left(x_{h}(\phi)\right)-V(\phi)\right] .
$$

Converse Lyapunov theorems and the construction of so-called complete-type Lyapunov-Krasovskii functionals are discussed in [4].

Imposing a particular structure on the functional, e.g., a form depending only a finite number of free parameters, often leads to easy-to-check stability criteria (for instance, in the form of LMIs), yet as price to pay the obtained results may be conservative in the sense that the sufficient stability conditions might not be close to necessary conditions. As an alternative to Lyapunov functionals, Lyapunov functions can be used as well, provided that the condition $\dot{V}<0$ is relaxed (the so-called Lyapunov-Razhumikhin approach), see, for example, [2].

More recent contributions on stability of systems with time-varying delay originate from a similar perturbation point of view, where the system is seen as a perturbation of a system with constant (possibly non-zero) delay.

\subsection{Delay differential equations as perturbation of ODEs}

Many results on stability, robust stability and control of time-delay systems are explicitly or implicitly based on a perturbation point of view, where delay differential equations are seen as perturbations of ordinary differential equations. For instance, in the literature a classification of stability criteria is often presented in terms of delay-independent criteria (conditions holding for all values of the delays) and delay-dependent criteria (usually holding for all delays smaller than a bound). This classification has its origin at two different ways of seeing (3) as a perturbation of an ODE, with as nominal system $\dot{x}(t)=A_{0} x(t)$ and $\dot{x}(t)=\left(A_{0}+A_{1}\right) x(t)$ (system for zero delay), respectively. This observation is illustrated in Figure 2 for results based on input-output and Lyapunov based approaches. 


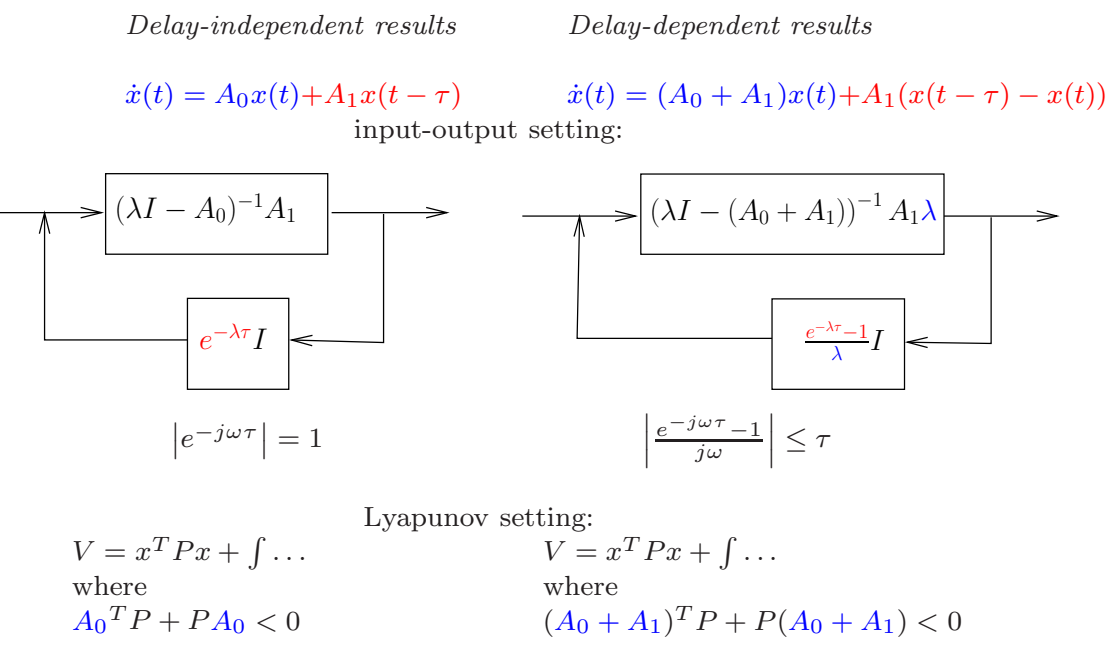

Fig. 2 The classification of stability criteria in delay-independent results and delaydependent results stems from two different perturbation viewpoints. Here, perturbation terms are printed in red.

\section{The spectrum of linear time-delay systems}

\subsection{Two eigenvalue problems}

The substitution of an exponential solution in (3) leads us to the nonlinear eigenvalue problem

$$
\left(\lambda I-A_{0}-A_{1} e^{-\lambda \tau}\right) v=0, \lambda \in \mathbb{C}, v \in \mathbb{C}^{n}, v \neq 0 .
$$

The solutions of the equation $\operatorname{det}\left(\lambda I-A_{0}-A_{1} e^{-\lambda \tau}\right)=0$ are called characteristic roots. Similarly, formulation (4) leads to the equivalent infinitedimensional linear eigenvalue problem

$$
(\lambda I-\mathcal{A}) u=0, \lambda \in \mathbb{C}, u \in \mathcal{C}\left([-\tau, 0], \mathbb{C}^{n}\right), u \not \equiv 0 .
$$

The combination of these two viewpoints lay at the basis of most methods for computing characteristic roots, see [6]. On the one hand, discretizing (7), i.e., approximating $\mathcal{A}$ with a matrix, and solving the resulting standard eigenvalue problems allows to obtain global information, for example, estimates of all characteristic roots in a given compact set or in a given right half plane. On the other hand, the (finitely many) nonlinear equations (6) allow to make local corrections on characteristic root approximations up to the desired accuracy, e.g., using Newton's method or inverse residual iteration. 
Linear time-delay systems satisfy spectrum determined growth properties of solutions. For instance, the zero solution of (3) is asymptotically stable if and only if all characteristic roots are in the open left half plane.

In Figure 3 (left), the rightmost characteristic roots of (2) are depicted for $k \tau=1$. Note that, since the characteristic equation can be written as $\lambda \tau+k \tau e^{-\lambda \tau}=0, k$ and $\tau$ can be combined into one parameter. In Figure 3 (right), we show the real parts of the characteristic roots as a function of $k \tau$. The plots illustrate some important spectral properties of retarded type FDEs. First, even though there are in general infinitely many characteristic roots, the number of them in any right half plane is always finite. Second, the individual characteristic roots, as well as the spectral abscissa, i.e., the supremum of the real parts of all characteristic roots, continuously depend on parameters. Related to this, a loss or gain of stability is always associated with characteristic roots crossing the imaginary axis. Figure 3 (right) also illustrates the transition to a delay-free system as $k \tau \rightarrow 0^{+}$.
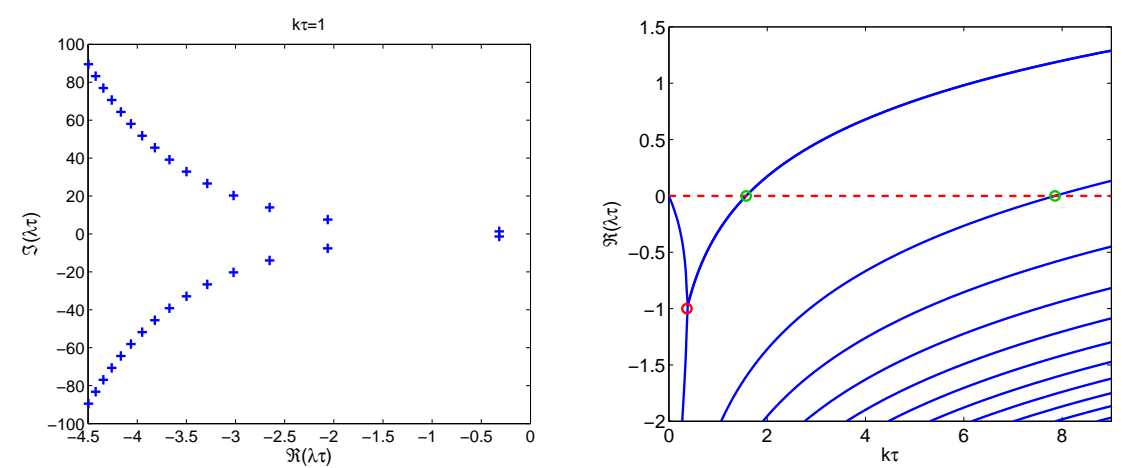

Fig. 3 (left) Rightmost characteristic roots of (2) for $k \tau=1$. (right) Real parts of rightmost characteristic roots as a function of $k \tau$.

\subsection{Critical delays: a finite dimensional characterization}

Assume that, for a given value of $k$, we are looking for values of the delay $\tau_{c}$ for which $(2)$ has a characteristic root $j \omega_{c}$ on the imaginary axis. From $j \omega=-k e^{-j \omega \tau}$ we get

$$
\omega_{c}=k, \tau_{c}=\frac{\frac{\pi}{2}+l 2 \pi}{\omega_{c}}, \quad l=0,1, \ldots, \quad \Re\left\{\left.\frac{d \lambda}{d \tau}\right|_{\left(\tau_{c}, j \omega_{c}\right)}\right\}^{-1}=\frac{1}{\omega_{c}^{2}} .
$$


Critical delay values $\tau_{c}$ are indicated with green circles on Figure 3 (right). The above formulas first illustrate an invariance property of imaginary axis roots and their crossing direction with respect to delay shifts of $2 \pi / \omega_{c}$. Second, the number of possible values of $\omega_{c}$ is one and thus finite. More generally, substituting $\lambda=j \omega$ in (6) and treating $\tau$ as a free parameter leads to a twoparameter eigenvalue problem

$$
\left(j \omega I-A_{0}-A_{1} z\right) v=0,
$$

with $\omega$ on the real axis and $z:=\exp (-j \omega \tau)$ on the unit circle. Most methods to solve such a problem boil down to an elimination of one of the independent variables $\omega$ or $z$. As an example of an elimination technique, we directly get from (9),

$$
\begin{aligned}
& j \omega \in \sigma\left(A_{0}+A_{1} z\right),-j \omega \in \sigma\left(A_{0}^{*}+A_{1}^{*} z^{-1}\right) \\
& \Rightarrow \operatorname{det}\left(\left(A_{0}+A_{1} z\right) \oplus\left(A_{0}^{*}+A_{1}^{*} z^{-1}\right)\right)=0 \\
& \Rightarrow \operatorname{det}\left(\left(A_{0} z+A_{1} z^{2}\right) \oplus\left(A_{0}^{*} z+A_{1}^{*}\right)\right)=0 .
\end{aligned}
$$

where $\sigma(\cdot)$ denotes the spectrum and $\oplus$ the Kronecker sum. Clearly, the resulting quadratic eigenvalue problem in $z$ is finite-dimensional.

\section{Control of time-delay system}

\subsection{Limitations induced by delays}

It is well known that delays in controls loop may lead to a significant degradation of performance and robustness and even to instability [9, 11]. Let us return to example (2). As illustrated with Figure 3 and expressions (8), the system loses stability if $\tau$ reaches the value $\pi / 2 k$, while stability cannot not be recovered for larger delays. The maximum achievable exponential decay rate of the solutions, which corresponds to the minimum of the spectral abscissa, is given by $-1 / \tau$, hence, large delays can only be tolerated at the price of a degradation of the rate of convergence. It should be noted that the limitations induced by delays are even more stringent if the uncontrolled systems is exponentially unstable, which is not the case for (2).

The analysis in the previous sections gives a hint why control is difficult in the presence of delays: the system is inherently infinite-dimensional. As a consequence, most control design problems which involve determining a finite number of parameters can be interpreted as reduced-order control design problems or as control design problems for under-actuated systems, which both are known to be hard problems. 


\subsection{Fixed-order control}

Most standard control design techniques lead to controllers whose dimension is larger or equal to the dimension of the system. For infinite-dimensional time-delay system such controllers might have a disadvantage of being complicated and hard to implement. To see this, for a system with delay in the state the generalization of static state feedback, $u(t)=k(x)$ is given by

$$
u(t)=\int_{-\tau}^{0} x(t+\theta) d \mu(\theta),
$$

where $\mu$ is a function of bounded variation. However, in the context of largescale systems it is know that reduced-order controllers often perform relative well compared to full order controllers, while they are much easier to implement.

Recently new methods for the design of controllers with a prescribed order (dimension) or structure have been proposed [6]. These methods rely on a direct optimization of appropriately defined cost functions (spectral abscissa, $\mathcal{H}_{2} / \mathcal{H}_{\infty}$ criteria). While $\mathcal{H}_{2}$ criteria can be addressed within a derivative based optimization framework, $\mathcal{H}_{\infty}$ criteria and the spectral abscissa require targeted methods for non-smooth optimization problems. To illustrate the need for such methods consider again Figure 3 (right): minimizing the spectral abscissa for a given value of $\tau$ as a function of the controller gain $k$ leads to an optimum where the objective function is not differentiable, even not locally Lipschitz, as shown by the red circle. In case of multiple controller parameters, the path of steepest descent in the parameter space typically has phases along a manifold characterized by the non-differentiability of the objective function.

\subsection{Using delays as controller parameters}

In contrast to the detrimental effects of delays, there are situations where delays have a beneficial effect and are even used as controller parameters, see [12]. For instance, delayed feedback can be used to stabilize oscillatory systems where the delay serves to adjust the phase in the control loop. An illustration is given in Figure 4, which depicts the stability regions of oscillator

$$
\ddot{x}(t)=-x(t)+u(t), y(t)=x(t)
$$

controlled with delayed output feedback, $u(t)=-k y(t-\tau)$. Note that for $\tau=$ 0 the (second order) system is not stabilizable by static output feedback. For small $k$, the sequence of stability - instability regions in the delay parameter space is related to the feedback acting in-phase or anti-phase with respect to 
the oscillation of the uncontrolled system. Note that, by taking $k$ sufficiently small, systems with arbitrarily large input delay can be stabilized as well.

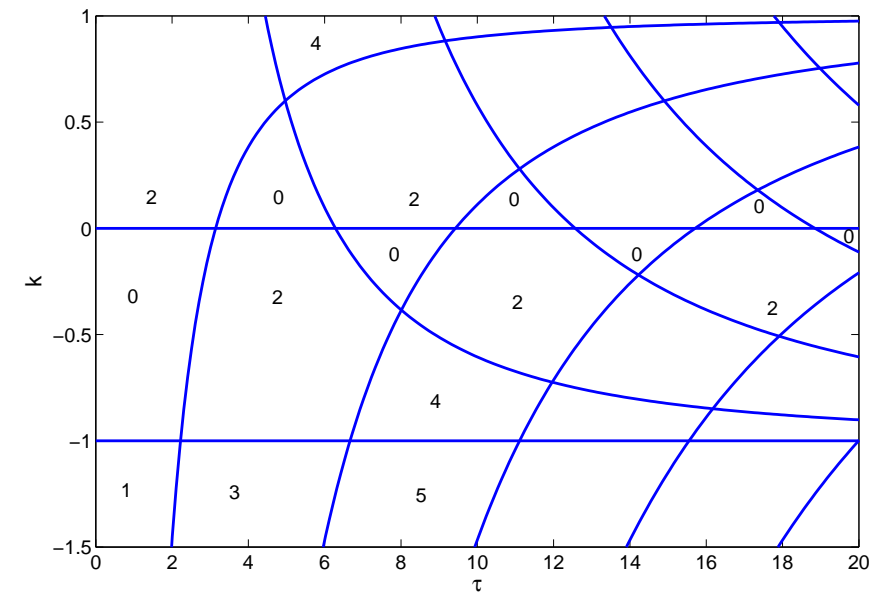

Fig. 4 Stability regions in the $(k, \tau)$ - parameter space of an oscillator controlled by delayed output feedback. The numbers refers to the number of characteristic roots in the open right half plane.

Delayed terms in control laws can also be used to approximate derivatives in the control action. For example a PI controller can by approximated by a control law of the form

$$
u(t)=-k_{1} y(t)-k_{2} \frac{y(t)-y(t-\tau)}{\tau},
$$

for $\tau$ sufficiently small. This principle can be extended towards higher order derivatives (combined with a scaling property it allows, for instance, to derive stabilizing control laws for integrator chains, using control laws of the form $u(t)=\sum_{i} k_{i} y\left(t-\tau_{i}\right)$ where the delays can be prescribed [8]). The experimental reconstruction and visualization of attractors of systems of high order via delayed time-series of the output is grounded in a similar idea.

Control laws of the form

$$
u(t)=F(y(t)-y(t-\tau))
$$

which depend on time-differences of state or output variables, so-called Pyragas type feedback, have the property that the position of equilibria and the shape of periodic orbits with period $\tau$ are not affected (since the control law vanishes whenever $y(t)=y(t-\tau)$ ), in contrary to their stability properties. 
Last but not least, delays can be used in control schemes to generate predictions or to stabilize predictors, which allow to compensate delays and improve performance $[15,5]$. Let us illustrate the main idea once more with system (1).

System (1) has a special structure, in the sense that the delay is only in the input, and it is advantageous to exploit this structure in the context of control. Coming back to the didactic example, the person who is taking a shower is possibly after some bad experiences- aware about the delay and will take into account his/her prediction of the system's reaction when adjusting the cold and hot water supply. Let us, to conclude, formalize this. The uncontrolled system can be rewritten as $\dot{x}(t)=v(t)$, where $v(t)=u(t-\tau)$. We know $u$ up to the current time $t$, thus we know $v$ up to time $t+\tau$ and, if $x(t)$ is also known, we can predict the value of $x$ at time $t+\tau$ :

$$
x_{p}(t+\tau)=x(t)+\int_{t}^{t+\tau} v(s) d s=x(t)+\int_{t-\tau}^{t} u(s) d s,
$$

and use the predicted state for feedback. With the control law $u(t)=$ $-k x_{p}(t+\tau)$ there is only one closed-loop characteristic root at $\lambda=-k$, i.e., as long as the model used in the predictor is exact, the delay in the loop is compensated by the prediction. For further reading on prediction based controllers, see, e.g., [5] and the references therein.

\section{From stand-alone systems to networks of interconnected systems}

We briefly discuss the effects of delays in networks of coupled systems, focusing on synchronization.

There are many examples of networks of interacting dynamical systems that exhibit collective behavior. The most unambiguous form of collective behavior is that of synchronization, which refers to the state in which all systems in the network behave identically. Fireflies emit their light pulses at the same instants in time; crickets chirp in unison for extended periods of time; and electrons move in synchrony in superconducting Josephson junctions [14].

Synchronization is a form of relative stability, i.e., a stability property of the zero solution of the error dynamics, which describe the differences between the state variables of the systems. A special case of synchronization problems are consensus problems, where the state variables of the different systems converge to a common steady state value. Consensus problem are also important in the context of distributed decision making, social networks (opinion dynamics), distributed and cooperative control (active cruise control, formation stabilization), just to mention a few [10]. 
Networks of identical diffusive delay-coupled systems are studied in depth in [13]. In order to highlight the effects of coupling delays, it suffices to analyze a model problem consisting of two coupled systems,

$$
\dot{x}_{i}(t)=f\left(x_{i}(t)\right)+u_{i}(t), \quad i=1,2,
$$

with coupling

$$
\left\{\begin{array}{l}
u_{1}(t)=k_{1}\left(x_{2}(t-\tau)-x_{1}(t)\right), \\
u_{2}(t)=k_{2}\left(x_{1}(t-\tau)-x_{2}(t)\right) .
\end{array}\right.
$$

The linear coupling functions (possibly with zero delay) as in (10) appear in a large number of applications, such as, networks of coupled neurons, networks of biological systems, coupled mechanical systems and electrical systems. The coupling signal $u_{i}(t)$ of node $i$ is (in general) defined as the sum of weighted differences of time-delayed outputs of connected systems and the node's own output at time instant $t, y_{i}(t)$. In this type of coupling the delay models the effect of finite speed of signal transmission.

Most nonlinear oscillators encountered in applications from engineering, physics and biology are semi-passive, meaning that they behave as passive systems sufficiently far from the origin. Semi-passivity implies boundedness of solutions of the coupled systems under mild additional conditions, also in the presence of delay.

The presence of observable synchronized behavior requires two properties to be satisfied: the presence of a forward invariant manifold characterized by synchronized motion, a so-called synchronization manifold, and (2) the satisfaction of a stability property of this manifold, in the sense that it attracts neighboring solutions. To clarify the role of delays, let us consider the above example and make a change of coordinate, $e(t)=x_{2}(t)-x_{1}(t)$, resulting in

$$
\left\{\begin{aligned}
\dot{x}_{1}(t)= & f\left(x_{1}(t)\right)+k_{1}\left(x_{1}(t)-x_{1}(t-\tau)\right)+k_{1} e(t), \\
\dot{e}(t)= & f\left(x_{1}(t)+e(t)\right)-f\left(x_{1}(t)\right)-k_{2} e(t)-k_{1} e(t-\tau) \\
& +\left(k_{1}-k_{2}\right)\left(x_{1}(t)-x_{1}(t-\tau)\right) .
\end{aligned}\right.
$$

In the delay free case (11) becomes

$$
\left\{\begin{array}{l}
\dot{x}_{1}(t)=f\left(x_{1}(t)\right)+k_{1} e(t) \\
\dot{e}(t)=f\left(x_{1}(t)+e(t)\right)-f\left(x_{1}(t)\right)-\left(k_{1}+k_{2}\right) e(t) .
\end{array}\right.
$$

Comparing (11) and (12) we conclude the following:

1. In the presence of delay, the existence of a partial synchronization manifold (i.e., $e \equiv 0$ being a solution of the second equation) requires $k_{1}=k_{2}$, whereas $e \equiv 0$ always solves the second equation of (12). This illustrates that additional structural requirements on the coupling may be necessary in the presence of delay.

2. Assuming $k_{1}=k_{2}$, the dynamics on the synchronization manifold of (11) are described by 


$$
\dot{x}_{1}(t)=f\left(x_{1}(t)\right)+k_{1}\left(x_{1}(t)-x_{1}(t .-\tau)\right),
$$

which can be interpreted as the dynamics of one system, controlled with Pyragas type time-delayed feedback. Since the dynamics on the synchronization manifold are affected by the coupling, we call the type of coupling (10) invasive for $\tau \neq 0$. On the other hand, the dynamics on the synchronization manifold of (12) are described by the dynamics of one individual system.

3. For $k_{1}=k_{2}$ the dynamics transversal to the synchronization manifold of (11), and its stability properties, are determined by the stability properties of an equilibrium of a delay differential equation with time-varying coefficients (considering $x_{1}$ as an exogenous signal), necessitating targeted analysis and synthesis tools. Local stability is described by the linearization

$$
\delta \dot{e}(t)=\frac{\partial f}{\partial x}\left(x_{1}(t)\right) \delta e(t)-k_{2} \delta e(t)-k_{1} \delta e(t-\tau) .
$$

In the case of more complex networks, a decoupling of the linearized error dynamic is possible based on the eigenstructure of the graph Laplacian matrix.

Remark 1. Besides (10) another frequency encountered coupling type is described by

$$
\left\{\begin{array}{l}
u_{1}(t)=k_{1}\left(x_{2}(t-\tau)-x_{1}(t-\tau)\right), \\
u_{2}(t)=k_{2}\left(x_{1}(t-\tau)-x_{2}(t-\tau)\right),
\end{array}\right.
$$

that is, both the node's own output and the outputs of connected systems are delayed by an amount of $\tau$. This type of coupling models the effects of sensor/actuator delay. Coupling (13) is non-invasive since it vanished on the synchronization manifold and, therefore, it does not affect the dynamics.

For large network, the emerging behavior in bifurcations may be very complex, and the error dynamics high dimensional and hard to analyze, but simplifies when the coupling strength is strong. Form more info we refer to [13] and the references therein.

Finally, let us take, instead of mutual coupling, a master-slave setting and make a connection with predictive feedback in Section 4.3. Suppose we want to predict the state of a possibly chaotic system

$$
\dot{x}(t)=f(x(t)),
$$

over a time-window of length $\tau$. To achieve this one can build an "observer" of the form

$$
\dot{z}(t)=f(z(t))+K(z(t-\tau)-x(t)) .
$$

The key idea is that the second, correction term vanishes on the moment that $x$ behaves as a delayed version of $z$. The prediction error $e(t)=z(t-\tau)-x(t)$ satisfies

$$
\dot{e}(t)=f(x(t)+e(t))-f(x(t))+K e(t-\tau) .
$$


The predictor is stable and the prediction reliable when $K$ and $\tau$ are such that the null solution of (14) is asymptotically stable. Hence, eventually the synthesis of the predictor boils down to a stabilization problem with delayed feedback, which is prone to the fundamental limitations sketched in Section 4.1. The above idea is elaborated in [3] and called anticipating synchronization.

\section{Conclusions}

Time-delay systems, which appear in a large number of applications, in particular in the context of networked systems, are a class of infinite-dimensional systems, resulting in rich dynamics and challenges from a control point of view. The different representations and interpretations and, in particular, the combination of viewpoints lead a wide variety of analysis and synthesis tools.

\section{Acknowledgments}

This work was supported by he project G0A5317N of the Research FoundationFlanders (FWO - Vlaanderen), and by the project UCoCoS, funded by the European Unions Horizon 2020 research and innovation programme under the Marie Sklodowska-Curie Grant Agreement No 675080.

\section{References}

1. A. Bellen and M. Zennaro. Numerical methods for delay differential equations. Oxford University Press, 2003.

2. K. Gu, V. L. Kharitonov, and J. Chen. Stability of time-delay systems. Birkhäuser, 2003.

3. H. Huijberts, H. Nijmeijer, and T. Oguchi. Anticipating synchronization of chaotic Lur'e systems. Chaos, 17(013117), 2007.

4. V. L. Kharitonov. Time-delay systems. Lyapunov functionals and matrices. Birkhäuser, 2013.

5. M. Krstic. Delay Compensation for nonlinear, adaptive, and PDE systems. Birkhauser, 2009.

6. W. Michiels. Design of fixed-order stabilizing and $\mathcal{H}_{2}-\mathcal{H}_{\infty}$ optimal controllers: An eigenvalue optimization approach. Lecture Notes in Control and Information Sciences, 423:201-216, 2012.

7. W. Michiels. Control of linear systems with delays. In J. Baillieul and T. Samad, editors, The Encyclopedia of Systems and Control. Springer Verlag, 2015.

8. W. Michiels and S.-I. Niculescu. Stability, control and computation for time-delay systems. An eigenvalue based approach. SIAM, 2 edition, 2014. 
9. S.-I. Niculescu. Delay effects on stability. A robust control approach, volume 269 of Lecture Notes in Control and Information Sciences. Springer Verlag, 2001.

10. R. Olfati-Saber and R. M. Murray. Consensus problems in networks of agents with switching topology and time-delays. IEEE Transactions on Automatic Control, 49(9):1520-1533, 2004.

11. J.-P. Richard. Time-delay systems: An overview of recent advances and open problems. Automatica, 39(10):1667-1694, 2003.

12. R. Sipahi, S. Niculescu, C. Abdallah, W. Michiels, and K. Gu. Stability and stabilization of systems with time delay. IEEE Control Systems Magazine, 31(1):38-65, 2011.

13. E. Steur, T. Oguchi, and H. Nijmeijer. Synchronization of systems with time-delayed coupling. World Scientific, 2016. In press.

14. S. H. Strogatz. Sync: the emerging science of spontaneous order. Hyperion, 1 edition, 2003.

15. Q.-C. Zhong. Robust control of time-delay systems. Springer Verlag: London, 2006. 